\title{
REGULARIZATION BY ERASEMENT
}

\author{
JUAN-ENRIQUE MARTÍNEZ-LEGAZ and JEAN-PAUL PENOT*
}

\begin{abstract}
We study an approximation method for sets and functions which erases corners but keeps smooth parts. Basic properties of such a method are pointed out in a general and simple way. Several convergence results are provided, essentially in the framework of variational analysis.
\end{abstract}

\section{Introduction}

Several regularization processes are known; all of them have advantages and drawbacks. Integral convolution with mollifiers is convenient for regularizing functions in finite dimensional spaces, in particular for the study of partial differential equations but is not of common use for optimization problems. The Moreau regularization is valid in any normed vector space and for any function with values in $R \cup\{+\infty\}$ and it has the advantage of preserving infima and the set of minimizers; thus it is widely used in optimization theory (see [26] for a general approach). However it requires the function to be bounded below by a function of quadratic decrease, and it yields a function the growth of which is at most quadratic, hence may be very different from the growth of the original function. These two weaknesses can be eliminated by considering a regularizing kernel of sufficiently strong growth (see [13], [41], for instance). Nonetheless it must be observed that the value of the function is usually changed at points of smoothness (while the value of the function at nonsmoothness points may be preserved, as in the case of the absolute value function on R). In particular, the regularized function of a regular function usually differs from the original function.

It is our purpose here to study a regularization process for sets and functions which leaves unchanged sets and functions which are regular enough. This

\footnotetext{
* The first author has been partially supported by the Ministerio de Ciencia y Tecnología and the FEDER project BEC2002-00642, and by the Comissionat per Universitats i Recerca de la Generalitat de Catalunya, grant SGR2001-00162. This work has been completed during a visit of the first author to the Laboratoire de Mathématiques Appliquées of the Université de Pau et des Pays de l'Adour, to which he is grateful for the support received. He also thanks the support of the Barcelona Economics Program of CREA. Both authors are indebted to C. Zălinescu for his careful reading and criticisms.
}

Received October 18, 2004. 
process is closely related to a double approximation procedure introduced in geometrical terms by Benoist ([7], [8]) following a method previously devised for functions by Lasry and Lions ([25]). However, as shown in the last section of the present paper, our process differs from the one of Benoist by several features, the main one being that our approximation sets are smaller than the original set, whereas the approximation sets of Benoist are larger. Our approximations are also closely related to the ones in Clarke, Stern and Wolenski [16] and Clarke, Ledyaev and Stern [14], [15]. In these references, as in the works of Cornet and Czarnecki [17], [18], [19], the authors stress the smoothness of the approximations of epi-Lipschitzian subsets of $\mathrm{R}^{n}$; here we do not make such an assumption and the space is a general Banach space. We give some attention to the case the set (or the function) is convex, as in [24], where a striking application of the rolling ball regularization method introduced by Seeger [47] is made to show the relationships between the main results of dynamic optimization. Our process is well suited for that case, but is inefficient for the case of continuous concave functions.

In the next section we present the general version of our approximation method; although the main case of interest is the case in which the "rounding" set $B$ is a ball, we devise basic properties valid with a general choice of this set. In particular, choosing for $B$ the epigraph of a quadratic function one would recover the Lasry-Lions method; one could also choose for $B$ the epigraph of a smooth convex function of different growth, in view of the observation made in [13, section 2] and completed in [1] that growth conditions are crucial. The study of this regularization procedure for functions is conducted in section 3 in which the links between the two processes are clarified. Various convergence results are displayed in section 4; although we reach convergence for the important Painlevé-Kuratowski, Wijsman, Mosco and bounded(-Hausdorff) convergences, the study is by no means comprehensive. For instance, we do not consider the strong convergence results of [17], [18], [19] in which one gets convergence of the normal cones. We devote section 5 to a short account of some algebraic aspects of our procedure. Some extensions and some comparisons with previous works are delineated in the last section.

In order to illustrate our method, one can imagine the regularized set corresponds to the place cleaned by some robot with a zone of action $B$ inside a room $E$; inasmuch $B$ is a round area, the corners of $E$ cannot be reached; but the smooth parts of the boundary are attained, especially if $B$ is small enough. The result is quite different from the one obtained while cleaning a polluted sea area $E$ by approaching it from the outside; in this second process the extreme points are approached but the cleaned area may be remote from the smooth parts of the polluted zone. 


\section{Regularization of sets}

Given a normed vector space $X$ and a nonempty subset $B$, we define the $B$ regularized set associated with a closed subset $E$ of $X$ as the set

$$
E_{B}:=\operatorname{cl}((E \boxminus B)+B),
$$

where $C+D$ denotes the Minkowski sum $\{c+d: c \in C, d \in D\}$, cl stands for closure and

$$
C \boxminus D:=\{x \in X: x+D \subset C\} .
$$

This last operation has been used by several authors (see [2], [34], [40] and their references). It enjoys useful properties. Among them we note that $C \boxminus D$ is convex when $C$ is convex and $C \boxminus D=C \boxminus \operatorname{cl}(D)$ when $C$ is closed; moreover, for each $x \in X$ one has $(x+E) \boxminus B=x+E \boxminus B$ and $E \boxminus(x+B)=-x+E \boxminus B$. In some favorable cases, the definition of $E_{B}$ can be simplified, and then one says that the regularization is exact when $E_{B}=E_{B}^{\prime}$ with $E_{B}^{\prime}:=(E \boxminus B)+B$, i.e. when $E_{B}^{\prime}$ is closed.

Lemma 2.1. One has $E_{B}=(E \boxminus B)+B$ when one of the following assumptions is fulfilled:

(a) $B$ is compact;

(b) $B$ is weakly compact and $E$ is weakly closed;

(c) $B$ and $E$ are convex, one of them is locally compact and their asymptotic cones satisfy $(-B)_{\infty} \cap E_{\infty}=\{0\}$ or, more generally, $(-B)_{\infty} \cap(E \boxminus$ $B)_{\infty}=\{0\}$.

Proof. The first two criteria obviously ensure that $(E \boxminus B)+B$ is closed. The third one is a special case of the Dieudonné criterion; it can be generalized to the nonconvex case by using the notion of asymptotic compactness (see [38]).

Let us display some elementary properties of our process.

PROPOSITION 2.2.

(a) If $E \subset F$, then one has $E_{B} \subset F_{B}$.

(b) If $B \subset A \subset \operatorname{cl}(B)$, then one has $E_{A}=E_{B}=\operatorname{cl}((E \boxminus B)+A)$.

(c) If $B+C \subset A \subset \operatorname{cl}(B+C)$, then one has $E_{A} \subset E_{B}$.

(d) If $B+C \subset A \subset \operatorname{cl}(B+C), B \subset A$ and $C \subset 0^{+} E:=E \boxminus E$ then $E_{A}=E_{B}$

(e) $(x+E)_{B}=x+E_{B}, E_{x+B}=E_{B}$.

(f) If $\lambda \neq 0$, then one has $(\lambda E)_{B}=\lambda E_{\frac{1}{\lambda} B}$.

(g) $E \boxminus F \subset E_{B} \boxminus F_{B}$.

(h) If $B$ and $E$ are convex, then $E_{B}$ is convex. 
In view of assertion (e) there is no loss of generality in assuming that $0 \in B$.

Proof. Assertions (a), (e), (f) and (h) are obvious, taking into account the properties of the operation $\boxminus$ mentioned above. Assertion (b) is a consequence of the equality $E \boxminus A=E \boxminus B$ when $E$ is closed and $B \subset A \subset \operatorname{cl}(B)$ and of the equality $\operatorname{cl}((E \boxminus B)+B)=\operatorname{cl}((E \boxminus B)+\operatorname{cl}(B))$.

To prove (c), since $E_{B}$ is closed and $A \subset \operatorname{cl}(B+C)$, it suffices to show that when $x \in E \boxminus A$ and $a=b+c$, with $b \in B, c \in C$ then one has $x+a \in E_{B}$. By assumption, for each $b^{\prime} \in B$ we have $x+b^{\prime}+c \in x+A \subset E$, so that $x+c \in E \boxminus B$. Then $x+a=(x+c)+b \in(E \boxminus B)+B \subset E_{B}$.

(d) Given $x \in E \boxminus B$, we have $x+B \subset E$, hence, by our assumption, $x+B+C \subset E$, so that $x \in E \boxminus A$ and $x+B \subset x+A \subset E_{A}$. Since $E_{A}$ is closed, we get $E_{B} \subset E_{A}$.

We shall now prove (g). If $x \in E \boxminus F$ then $x+F \subset E$ whence, by (c) and (a), $x+F_{B}=(x+F)_{B} \subset E_{B}$.

Notice that $E_{B} \subset E$, so that, in contrast with most existing regularization notions, our regularized sets are inner approximations. When $B$ is solid (i.e. such that $B$ is the closure of $\operatorname{int}(B)$ ), the preceding inclusion can be strengthened:

Proposition 2.3. If $B$ is solid, then one has $E_{B} \subset \operatorname{cl}(\operatorname{int}(E))$.

Proof. If $x \in(E \boxminus B)+B$ then $x-b \in E \boxminus B$ for some $b \in B$; thus

$$
\begin{gathered}
x \in x-b+B=x-b+\operatorname{cl}(\operatorname{int}(B))=\operatorname{cl}(\operatorname{int}(x-b+B)) \\
\subset \operatorname{cl}(\operatorname{int}((E \boxminus B)+B)) \subset \operatorname{cl}(\operatorname{int}(E)) .
\end{gathered}
$$

Thus, when $x \in E_{B}:=\operatorname{cl}((E \boxminus B)+B)$, one also has $x \in \operatorname{cl}(\operatorname{int}(E))$.

The preceding result shows that when $B$ is solid, the $B$-regularization is useless for sets with an empty interior. One may wonder whether one can get some relativization of the preceding proposition when $B$ is relatively solid in the sense that $B$ is the closure of its interior with respect to the relative topology of aff $(B)$, the smallest affine subspace containing $B$. The following example shows that one cannot expect that $E_{B} \subset \operatorname{cl}\left(\operatorname{int}_{E-B+\operatorname{aff}(B)}(E)\right)$.

ExAmple. Let $X:=\mathrm{R}^{2}, B:=[-1,1] \times\{0\}, E:=[-1,1]^{2} \cup[1,2]$. Then $E_{B}=E$ but $\operatorname{cl}\left(\operatorname{int}_{E-B+\operatorname{aff}(B)}(E)\right)=[-1,1]^{2}$.

REMARK. However one has the inclusion $E_{B} \subset \operatorname{cl}\left(\bigcup_{M \in M(B)} \operatorname{int}_{M}(E \cap M)\right)$, where $\mathcal{M}(B)$ denotes denotes the family of translates $x+\operatorname{aff}(B)$ as $x \in X$ (or even $x \in E \boxminus B$, yielding the smaller family $\mathcal{M}(B, E)$ ). To see that, let 
$x \in E_{B}$. There exists some $b \in B$ such that $y:=x-b \in E \boxminus B$; then $x \in M:=y+\operatorname{aff}(B) \in M(B, E)$. Thus

$$
x \in y+B \subset y+\operatorname{cl}\left(\operatorname{int}_{\operatorname{aff}(B)}(B)\right)=\operatorname{cl}\left(\operatorname{int}_{y+\operatorname{aff}(B)}(y+B)\right) .
$$

Since $(y+B) \cap M \subset E \cap M$, we get $x \in \operatorname{cl}\left(\operatorname{int}_{M}(E \cap M)\right)$.

The fixed points of the $B$-regularization operator will be called $B$-regular subsets:

Definition 2.4. A closed set $E \subset X$ is said to be $B$-regular if $E_{B}=E$. It is said to be exactly $B$-regular if $E_{B}^{\prime}:=(E \boxminus B)+B=E$.

From Proposition 2.3 it follows that, if $B$ is solid, every $B$-regular set $E$ is solid too.

Proposition 2.5. $E$ is $B$-regular if and only if there exists a set $C$ such that $\operatorname{cl}(C+B)=E$.

Proof. To prove the "only if" part, take $C=E \boxminus B$. Conversely, assume that there exists a set $C$ such that $\operatorname{cl}(C+B)=E$. Clearly, $C \subset E \boxminus B$. Therefore, one has

$$
E=\operatorname{cl}(C+B) \subset \operatorname{cl}((E \boxminus B)+B)=E_{B} \subset E,
$$

so that $E$ is $B$-regular.

COROLlaRY 2.6. The B-regularization operator is idempotent: $\left(E_{B}\right)_{B}=$ $E_{B}$ for every closed subset $E$ of $X$.

Proof. By Proposition 2.5, the set $E_{B}$ is $B$-regular.

Corollary 2.7. $E_{B}$ is the largest $B$-regular subset of $E$.

Proof. We already know that $E_{B}$ is a $B$-regular subset of $E$; if $F \subset E$ is $B$-regular then, by Proposition 2.2 (a), one has $F=F_{B} \subset E_{B}$.

Corollary 2.8. If $A=B+C$ and $E$ is $A$-regular, then $E$ is $B$-regular, too.

Proof. This follows from the inclusion $E_{B} \subset E$, combined with Proposition 2.2 (c): $E=E_{A} \subset E_{B} \subset E$.

A characterization of $B$-regularity can be deduced from the last proposition by using support functions. Recall that the support function of a subset $E$ of $X$ is the function $\sigma_{E}$ on $X^{*}$ given by $\sigma_{E}\left(x^{*}\right):=\sup \left\{\left\langle x^{*}, x\right\rangle: x \in E\right\}$. 
COROllary 2.9. If $B$ is bounded and convex, a nonempty closed convex set $E$ is $B$-regular if and only if the function $\sigma_{E}-\sigma_{B}$ is convex, weak ${ }^{*}$ lower semicontinuous and does not take the value $-\infty$.

Proof. If $E$ is $B$-regular then $\operatorname{cl}((E \boxminus B)+B)=E$, whence $\sigma_{E \boxminus B}+\sigma_{B}=$ $\sigma_{E}$, so that $\sigma_{E}-\sigma_{B}$ is the convex function $\sigma_{E \boxminus B}$ which is lower semicontinuous and does not take the value $-\infty$ as $E \boxminus B$ is nonempty. Conversely, if $B$ is bounded and convex and if $\sigma_{E}-\sigma_{B}$ is convex, weak* lower semicontinuous and does not take the value $-\infty$, then, as it is also positively homogeneous, one has $\sigma_{E}-\sigma_{B}=\sigma_{C}$ for some closed convex set $C$; from this equality it follows that $\operatorname{cl}(C+B)=E$ and hence, by Proposition 2.5, $E$ is $B$-regular.

In the sequel, we will be mostly interested in the case $B:=\varepsilon B_{X}$, where $\varepsilon$ is a positive number and $B_{X}$ is the closed unit ball of $X$. To simplify notation and terminology, we set $E_{\varepsilon}:=E_{\varepsilon B_{X}}$ and shall use the terms $\varepsilon$-regularized and $\varepsilon$-regular instead of $\varepsilon B_{X}$-regularized and $\varepsilon B_{X}$-regular, respectively. The following proposition summarizes the specialization of the basic properties we have proved so far to this specific context:

PRoposition 2.10.

(a) If $E \subset F$, then one has $E_{\varepsilon} \subset F_{\varepsilon}$.

(b) If $\varepsilon \geq \varepsilon^{\prime}>0$ then $E_{\varepsilon} \subset E_{\varepsilon^{\prime}}$.

(c) $(x+E)_{\varepsilon}=x+E_{\varepsilon}$.

(d) If $\lambda \neq 0$ then $(\lambda E)_{\varepsilon}=\lambda E_{\frac{\varepsilon}{|\lambda|}}$.

(e) $E \boxminus F \subset E_{\varepsilon} \boxminus F_{\varepsilon}$.

(f) $E$ is $\varepsilon$-regular if and only if there exists a subset $C$ of $X$ such that $\operatorname{cl}\left(C+\varepsilon B_{X}\right)=E$. Thus $E$ is $\varepsilon$-regular if and only if $E$ is the closure of a union of balls of radius $\varepsilon$.

(g) $\left(E_{\varepsilon}\right)_{\varepsilon}=E_{\varepsilon}$.

(h) $E_{\varepsilon}$ is the largest $\varepsilon$-regular subset of $E$.

(i) If $\varepsilon \geq \varepsilon^{\prime}>0$ and $E$ is $\varepsilon$-regular then it is $\varepsilon^{\prime}$-regular, too.

Corollary 2.11. $\left(E_{\varepsilon}\right)_{\varepsilon^{\prime}}=E_{\varepsilon \vee \varepsilon^{\prime}}$.

Proof. We use properties (a), (b), (g) and (h) of Proposition 2.10. By the inclusion $E_{B} \subset E$, one has $\left(E_{\varepsilon}\right)_{\varepsilon^{\prime}} \subset E_{\varepsilon} \subset E$. From this last inclusion, using (a), it follows that $\left(E_{\varepsilon}\right)_{\varepsilon^{\prime}} \subset E_{\varepsilon^{\prime}}$. Thus $\left(E_{\varepsilon}\right)_{\varepsilon^{\prime}} \subset E_{\varepsilon} \cap E_{\varepsilon^{\prime}}=E_{\varepsilon \vee \varepsilon^{\prime}}$ by (b). To prove the opposite inclusion, we consider two cases: $\varepsilon \leq \varepsilon^{\prime}$ or $\varepsilon^{\prime} \leq \varepsilon$. If $\varepsilon \leq \varepsilon^{\prime}$ then, by (b), $E_{\varepsilon^{\prime}} \subset E_{\varepsilon}$, whence, using (g) and (a), one gets $E_{\varepsilon \vee \varepsilon^{\prime}}=E_{\varepsilon^{\prime}}=$ $\left(E_{\varepsilon^{\prime}}\right)_{\varepsilon^{\prime}} \subset\left(E_{\varepsilon}\right)_{\varepsilon^{\prime}}$. If $\varepsilon^{\prime} \leq \varepsilon$ then, by $(\mathrm{g})$ and $(\mathrm{b}), E_{\varepsilon \vee \varepsilon^{\prime}}=E_{\varepsilon}=\left(E_{\varepsilon}\right)_{\varepsilon} \subset\left(E_{\varepsilon}\right)_{\varepsilon^{\prime}}$.

Let us note the following consequence of assertion (f) of Proposition 2.10. It stems from the equality $\operatorname{cl}\left(\bigcup_{i \in I} \operatorname{cl}\left(C_{i}+\varepsilon B_{X}\right)\right)=\operatorname{cl}\left(\bigcup_{i \in I} C_{i}+\varepsilon B_{X}\right)$. 
COROLlary 2.12. The closure of the union of a family of $\varepsilon$-regular subsets of $X$ is $\varepsilon$-regular.

Corollary 2.13. Any solid subset $E$ of $X$ whose complement $E^{c}$ is convex is $\varepsilon$-regular for any $\varepsilon>0$.

Proof. Since $E$ is solid and since by the Hahn-Banach theorem int $E$ is the union of a family of closed half-spaces, using Corollary 2.12 it suffices to prove the result for a closed half-space. Let $E=h^{-1}\left(\mathrm{R}_{+}\right)$, where $h \in X^{*}$ has norm 1 . Let $\left(u_{n}\right)$ be a sequence of unit vectors such that $\left(h\left(u_{n}\right)\right) \rightarrow 1$. Given $\varepsilon>0$ and $x \in$ int $E$, let $y_{n}:=x+\varepsilon u_{n}$. Then $x \in y_{n}+\varepsilon B_{X}$ and for any $w \in y_{n}+\varepsilon B_{X}$ and any $n$ large enough, we have

$$
h(w) \geq h\left(y_{n}\right)-\varepsilon=h(x)+\varepsilon h\left(u_{n}\right)-\varepsilon>0
$$

since $h(x)>0$. Thus $y_{n}+\varepsilon B_{X} \subset$ int $E$ and $E=\operatorname{cl}($ int $E)$ is the closure of a union of $\varepsilon$-balls.

The following results confirm the remark following the proof of Proposition 2.3.

Proposition 2.14. Setting $E_{\varepsilon}^{\prime}:=\left(E \boxminus \varepsilon B_{X}\right)+\varepsilon B_{X}$, one has

$$
\operatorname{int}(E) \subset \bigcup_{\varepsilon>0} E_{\varepsilon}^{\prime} \subset \bigcup_{\varepsilon>0} E_{\varepsilon} \subset \operatorname{cl}(\operatorname{int}(E)) .
$$

Proof. For any $x \in \operatorname{int}(E)$ there exists $\varepsilon>0$ such that $x+\varepsilon B_{X} \subset E$, that is, $x \in E \boxminus \varepsilon B_{X}$; thus one has $x=x+\varepsilon 0 \in\left(E \boxminus \varepsilon B_{X}\right)+\varepsilon B_{X} \subset E_{\varepsilon}$. This proves the first inclusion. The last one follows from Proposition 2.3.

Corollary 2.15. For any closed subset $E$ of $X$ one has

$$
\operatorname{cl}\left(\bigcup_{\varepsilon>0} E_{\varepsilon}\right)=\operatorname{cl}\left(\bigcup_{\varepsilon>0} E_{\varepsilon}^{\prime}\right)=\operatorname{cl}(\operatorname{int}(E)) .
$$

Moreover, if $E$ is solid one has

$$
\operatorname{int}\left(\bigcup_{\varepsilon>0} E_{\varepsilon}\right)=\operatorname{int}\left(\bigcup_{\varepsilon>0} E_{\varepsilon}^{\prime}\right)=\operatorname{int}(E) .
$$

Corollary 2.16. $E_{\varepsilon} \neq \emptyset$ for some $\varepsilon>0$ if and only if $\operatorname{int}(E) \neq \emptyset$.

For a convex set $B$, the $B$-regularization has the nice property of preserving convexity, as seen in Proposition $2.2(\mathrm{~h})$; in the case of balls centered at 0 of arbitrary small radius, more can be said. 
Proposition 2.17. If $E$ is convex then $E_{\varepsilon}$ is convex for every $\varepsilon$. Conversely, if all the sets $E_{\varepsilon}$ are convex then $\operatorname{int}(E)$ is convex, hence $E$ is convex if it is solid.

Proof. The first assertion being obvious, we shall only prove the second one. If $E_{\varepsilon}$ is convex for every $\varepsilon$ then, by Proposition $2.10(\mathrm{~b})$, the set $\bigcup_{\varepsilon>0} E_{\varepsilon}$ is convex, whence, in view of Corollary 2.15, $\operatorname{int}(E)$ is convex.

The following result is a consequence of Corollary 2.9, since the support function of $\varepsilon B_{X}$ is $\varepsilon\|\cdot\|_{X^{*}},\|\cdot\|_{X^{*}}$ denoting the dual norm.

Proposition 2.18. A closed convex set $E$ is $\varepsilon$-regular if and only if the function $\sigma_{E}-\varepsilon\|\cdot\|_{X^{*}}$ is convex and weak* lower semicontinuous.

When $X$ is reflexive, $\sigma_{E}-\varepsilon\|\cdot\|_{X^{*}}$ is weakly lower semicontinuous whenever it is convex (as it is lower semicontinuous).

Corollary 2.15 yields a partial description of the sets $E^{\prime}:=\bigcup_{\varepsilon>0} E_{\varepsilon}^{\prime}$ and $\bigcup_{\varepsilon>0} E_{\varepsilon}$. Since $\operatorname{int}(E) \subset E^{\prime} \subset \bigcup_{\varepsilon>0} E_{\varepsilon} \subset E$, to give a full description one just has to characterize those boundary points of $E$ that belong to $E^{\prime}$. This will be done in the following proposition. We will use the following notion of $\varepsilon$-(metrically) normal set to $S$ at $x \in S$ (or $\varepsilon$-perpendicular set to $S$ at $x$ ), with $\varepsilon \in \mathbf{R}_{+}$:

$$
\begin{aligned}
N_{\varepsilon}(S, x) & =\{u \in X:\|u\|=\varepsilon=d(x+u, S)\} \\
& =\left\{u \in X:\|u\|=\varepsilon, \operatorname{int}\left(x+u+\varepsilon B_{X}\right) \cap S=\emptyset\right\},
\end{aligned}
$$

with $d(w, S):=\min \{\|w-s\|: s \in S\}$ for $w \in X$. Let us recall from [11], [21], [36] that the metrically normal cone to $S \subset X$ at $x \in S$ is

$$
\begin{aligned}
N_{m}(S, x) & =\{\lambda(w-x): \lambda \geq 0, w \in X,\|w-x\|=d(w, S)\} \\
& =\bigcup_{\varepsilon \geq 0} N_{\varepsilon}(S, x) .
\end{aligned}
$$

This cone is related to the proximal normal cone to $S \subset X$ at $x \in S$ which is the subset of $X^{*}$ given by $J\left(N_{m}(S, x)\right)$, where $J: X \rightrightarrows X^{*}$ is the duality map given by

$$
J(x):=\left\{x^{*}: x^{*} \in X^{*},\left\langle x^{*}, x\right\rangle=\|x\|^{2},\left\|x^{*}\right\|=\|x\|\right\} .
$$

In particular, when $X$ is a Hilbert space identified with its dual $X^{*}, N_{m}(F, x)$ coincides with the set of proximal normals.

Proposition 2.19. Let $x$ be a boundary point of $E$. Then $x \in E_{\varepsilon}^{\prime}:=$ $\left(E \boxminus \varepsilon B_{X}\right)+\varepsilon B_{X}$ with $\varepsilon>0$ if and only if $N_{\varepsilon}(\operatorname{cl}(X \backslash E), x) \neq \emptyset$. Thus $x \in E^{\prime}:=\bigcup_{\varepsilon>0} E_{\varepsilon}^{\prime}$ if and only if $N_{m}(\operatorname{cl}(X \backslash E), x) \neq\{0\}$. 
Proof. Let $x$ be a boundary point of $E$. Suppose first that $x \in E_{\varepsilon}^{\prime}:=$ $\left(E \boxminus \varepsilon B_{X}\right)+\varepsilon B_{X}$ for some $\varepsilon>0$. Then $x-\varepsilon b \in E \boxminus \varepsilon B_{X}$ for some $b \in B_{X}$. Hence, $x-\varepsilon b+\varepsilon B_{X} \subset E$, so that $d(x-\varepsilon b, \operatorname{cl}(X \backslash E)) \geq \varepsilon \geq\|x-\varepsilon b-x\| \geq$ $d(x-\varepsilon b, \operatorname{cl}(X \backslash E))$. We thus have $\|(x-\varepsilon b)-x\|=d(x-\varepsilon b, \operatorname{cl}(X \backslash E))=\varepsilon$, which implies $-\varepsilon b \in N_{\varepsilon}(\operatorname{cl}(X \backslash E), x)$.

Conversely, suppose that there exists some $u \in N_{\varepsilon}(\operatorname{cl}(X \backslash E), x):\|u\|=\varepsilon$ and $\operatorname{int}\left(x+u+\varepsilon B_{X}\right) \subset E$. Then, $E$ being closed, one has $x+u+\varepsilon B_{X} \subset E$, so that $x+u \in E \boxminus \varepsilon B_{X}$; hence $x=x+u-u \in\left(E \boxminus \varepsilon B_{X}\right)+\varepsilon B_{X}=E_{\varepsilon}^{\prime}$.

COROLlary 2.20. A boundary point $x$ of $E$ belongs to $E_{\varepsilon}$ whenever

$$
\limsup _{x^{\prime}(\in \operatorname{bd}(E)) \rightarrow x} N_{\varepsilon}\left(\operatorname{cl}(X \backslash E), x^{\prime}\right) \neq \emptyset .
$$

Proof. Let $x$ be a boundary point of $E$ such that (1) holds. Then there exist some sequences $\left(x_{n}\right) \rightarrow x$ in $\operatorname{bd}(E)$ and $\left(u_{n}\right) \rightarrow u \neq 0$ in $X$ with $u_{n} \in N_{\varepsilon}\left(\operatorname{cl}(X \backslash E), x_{n}\right)$ for each $n \in \mathrm{N}$. Then $x_{n} \in E_{\varepsilon}^{\prime}$ hence $x \in E_{\varepsilon}$.

In order to study the regularizing effect of the operator $E \mapsto E_{B}$, let us introduce some terminology. It uses the classical definition of tangent cone to a subset $S$ at some point $x \in S$ which is the set $T(S, x)$ of vectors $v \in X$ such that there exist sequences $\left(v_{n}\right) \rightarrow v,\left(t_{n}\right) \searrow 0$ satisfying $x+t_{n} v_{n} \in S$ for each $n \in \mathrm{N}$. When $S$ is convex, $T(S, x)=\operatorname{cl}\left(\mathrm{R}_{+}(S-x)\right)$. The normal cone $N(S, x)$ to $S$ at $x \in S$ is the polar cone of $T(S, x)$. It is easy to show that if the norm of $X$ is Gâteaux-differentiable off 0 , then $N(S, x)$ contains the proximal normal cone $J\left(N_{m}(S, x)\right)$ ). The interior tangent cone (or cone of interior displacements, in the terminology of [20]) to $S$ at $x$ is the set $I(S, x)$ of vectors $v \in X$ such that there exists $\varepsilon>0$ for which $x+(0, \varepsilon)\left(v+\varepsilon B_{X}\right) \subset S$. It is easy to see that

$$
I(S, x)=X \backslash T(X \backslash S, x) .
$$

In the following definition, we use the expression "half-space" to mean an homogeneous half-space $[f>0]$ or $[f \geq 0]$ defined by a continuous linear form $f$; in fact, in view of homogeneity arguments, the involved containments are not affected by this abuse of language.

Definition 2.21. A closed subset $S$ of $X$ is said to be blunt (resp. one-sided) at some boundary point $x$ of $S$ if the interior tangent cone (resp. the tangent cone) to $S$ at $x$ contains (resp. is contained in) some open (resp. closed) halfspace. A closed subset $S$ of $X$ is said to be blunt (resp. one-sided) if at each boundary point $x$ of $S$ it is blunt (resp. one-sided).

If $S$ is both blunt and one-sided (at some point $x \in S$ ), it is said to be smooth (at $x$ ). 
Thus, $S$ is blunt at $x$ if and only if $X \backslash S$ is one-sided at $x$. If $S$ is one-sided at some boundary point $x$ of $S$ then the normal cone $N(S, x)$ contains some half-line. Any convex set $S$ with a nonempty interior is one-sided; thus it is smooth at $x \in S$ if and only if it is blunt at $x$. The Farkas lemma ensures that when a set $S$ is smooth at $x$, the normal cone $N(S, x)$ is a half-line and $T(S, x)$ is a half-space whose interior is $I(S, x)$.

Proposition 2.22. If $B$ is blunt, then $E_{B}$ is blunt at each boundary point of $E_{B}$ belonging to $E_{B}^{\prime}:=(E \boxminus B)+B$.

In particular, if $E$ is convex and $B$ is convex, solid and smooth and if $E_{B}^{\prime}:=(E \boxminus B)+B$ is closed, then $E_{B}$ is smooth.

Proof. Let $x \in E_{B}^{\prime}$ be a boundary point of $E_{B}$ and let $b \in B$ be such that $y:=x-b \in E \boxminus B$. Hence $x=y+b \in y+B \subset(E \boxminus B)+B \subset E_{B}$ and therefore $x$ is a boundary point of $y+B$. If $H$ is a half-space contained in $I(B, b)$ one has

$$
H \subset I(B, b)=I(y+B, y+b) \subset I\left(E_{B}, x\right) .
$$

The second assertion follows from the fact that, under its assumptions, the set $E_{B}$ is convex with a non empty interior, so that the smoothness of $E_{B}=E_{B}^{\prime}$ is a consequence of the fact that it is blunt.

COROLlaRY 2.23. If $B$ is convex, weakly compact, solid and smooth then every $B$-regular convex set is smooth.

Since $B_{X}$ is smooth when the norm of $X$ is smooth off 0 (the Slater condition being automatically satisfied), we get the following special case of the proposition.

COROLlary 2.24. If $X$ is reflexive and if the norm of $X$ is Gâteaux differentiable on $X \backslash\{0\}$, then for any weakly closed subset $E$ of $X$ and for any $\varepsilon>0$ the set $E_{\varepsilon}$ is blunt and for any closed convex subset $E$ of $X$ the set $E_{\varepsilon}$ is smooth.

Next we are going to prove that, in the Euclidean space case, for a convex set, $\varepsilon$-regularity is equivalent to a weak convexity property of its complement. We need some definitions, in particular an extension of the notion of weak convexity with respect to $\varepsilon>0$ of [51] and the notion of $\varepsilon$-proximinal set (or set of positive reach in [21]); we will also use a combination of these two notions used in [16, Thm. 4.1].

DEFINITION 2.25.

(a) A closed subset $F$ of a n.v.s. $X$ is $\varepsilon$-weakly convex if for any $x$ in the boundary bd $(F)$ of $F$ one has $N_{\varepsilon}(F, x)=N_{m}(F, x) \cap \varepsilon B_{X}$, i.e. if for any $u \in N_{m}(F, x) \cap \varepsilon B_{X}$ one has $\operatorname{int}\left(x+u+\|u\| B_{X}\right) \cap F=\emptyset$. 
(b) It is said to be philonormal if for any $x$ in the boundary bd( $F)$ of $F$ the metrically normal cone to $F$ at $x$ is not reduced to $\{0\}$.

(c) It is said to be $\varepsilon$-proximinal if for any $x \in X$ such that $d(x, F)<\varepsilon$ there exists some $y \in F$ such that $\|x-y\|=d(x, F)$.

(d) It is $\varepsilon$-proximally smooth if it is both $\varepsilon$-proximinal and $\varepsilon$-weakly convex.

Condition (c) is obviously satisfied when $F$ is a weakly closed subset of a reflexive Banach space, in particular when $F$ is a closed subset of a finite dimensional space. The notion of $\varepsilon$-proximally smooth subset corresponds to the notion of proximally smooth subset for a specific radius $\varepsilon$ introduced and characterized in [16, Thm. 4.1] when $X$ is a Hilbert space. In [16, Cor. 4.15 and Remark 4.16] it is proved that when $X$ is a finite dimensional Hilbert space, any $\varepsilon$-proximally smooth subset is philonormal. In infinite dimensional Hilbert spaces one can find closed convex subsets which are not philonormal (see [12], [37, Example 3.1]). However, in any Hilbert space, any solid convex subset is philonormal. In finite dimensional Hilbert spaces, a characterization of $\varepsilon$-weakly convex subsets has been given in [51]: introducing for $\varepsilon>0$ the $\varepsilon$-lens of $x_{1}, x_{2} \in X$ by

$$
D_{\varepsilon}\left(x_{1}, x_{2}\right)=\bigcap_{x \in\left(x_{1}+\varepsilon B_{X}\right) \cap\left(x_{2}+\varepsilon B_{X}\right)}\left(x+\varepsilon B_{X}\right) .
$$

(which is well defined, i.e., the intersection is over a nonempty family) if and only if $\left\|x_{1}-x_{2}\right\| \leq 2 \varepsilon$, in which case $\left.\left[x_{1}, x_{2}\right] \subset D_{\varepsilon}\left(x_{1}, x_{2}\right)\right)$ it is proved in [51, Prop. 3.5] that $F$ is $\varepsilon$-weakly convex if and only if

$$
\begin{aligned}
D_{\varepsilon}\left(x_{1}, x_{2}\right) \cap F \neq & \left\{x_{1}, x_{2}\right\} \\
& \text { for any } x_{1}, x_{2} \in F \text { with } x_{1} \neq x_{2} \text { and }\left\|x_{1}-x_{2}\right\|<2 \varepsilon .
\end{aligned}
$$

Using [51, Prop. 3.4], one can easily check that a closed subset is convex if and only if it is $\varepsilon$-weakly convex for any $\varepsilon>0$. An example of a nonconvex $\varepsilon$-weakly convex set is the complement of any open ball with radius $\varepsilon>0$ [51, Prop. 3.1] in an Euclidean space.

TheOREm 2.26. Let $E$ be a solid subset of a n.v.s. $X$. If $F:=\operatorname{cl}(X \backslash E)$ is $\varepsilon$-proximally smooth and philonormal, then $E$ is exactly $\varepsilon$-regular.

Conversely, if a closed subset $E$ of $X$ is exactly $\varepsilon$-regular, then $E$ is solid and the closure $F:=\mathrm{cl}(X \backslash E)$ of its complement is philonormal. If moreover $E$ is one-sided, and if $X$ is smooth and strictly convex, then $F$ is $\varepsilon$-weakly convex. If moreover $X$ is reflexive and $F$ is weakly closed, $F$ is $\varepsilon$-proximally smooth. 
Proof. Assume first that $F:=\operatorname{cl}(X \backslash E)$ is $\varepsilon$-weakly convex, $\varepsilon$-proximinal and philonormal and let $x \in E$. If $x+\varepsilon B_{X} \subset E$ then $x \in E \boxminus \varepsilon B_{X} \subset E_{\varepsilon}$. Suppose now that $x+\varepsilon B_{X} \not \subset E$. Then there exists some $z \in X \backslash E \subset \operatorname{int}(F)$ such that $\|x-z\| \leq \varepsilon$; it follows that $\alpha:=d(x, F)<\varepsilon$. As $F$ is $\varepsilon$-proximinal, there exists $y \in F$ such that $\|x-y\|=\alpha$. Let us first consider the case $\alpha>0$; then $y \in \operatorname{bd}(F)$ and one has $x-y=\alpha u$, with $u \in N_{m}(F, y)$ and $\|u\|=1$. Since $F:=\operatorname{cl}(X \backslash E)$ is $\varepsilon$-weakly convex, for $w:=y+\varepsilon u$, we have $\operatorname{int}\left(w+\varepsilon B_{X}\right) \cap \operatorname{cl}(X \backslash E)=\emptyset$, hence $\left(w+\varepsilon B_{X}\right) \cap(X \backslash E)=\emptyset$ and $w \in E \boxminus \varepsilon B_{X}$. Then we have $x=w-(\varepsilon-\alpha) u \in\left(E \boxminus \varepsilon B_{X}\right)+\varepsilon B_{X}=E_{\varepsilon}^{\prime}$. When $\alpha=0$, we have $y=x \in F$; as $E$ is solid, we cannot have $x \in \operatorname{int}(F)$, since otherwise we would have $\operatorname{int}(F) \cap \operatorname{cl}(\operatorname{int}(E)) \neq \emptyset$, hence $\operatorname{int}(F) \cap \operatorname{int}(E) \neq \emptyset$, a fortiori $\operatorname{cl}(X \backslash E) \cap \operatorname{int}(E) \neq \varnothing$ and $(X \backslash E) \cap \operatorname{int}(E) \neq \varnothing$, a contradiction. Hence $x \in \operatorname{bd}(F)$. Then, as $F$ is philonormal, we can find $u \in N_{m}(F, x) \cap \varepsilon B_{X}$, $u \neq 0$. As above, setting $w:=x+\varepsilon u$, we get that $\operatorname{int}\left(w+\varepsilon B_{X}\right) \cap \operatorname{cl}(X \backslash E)=\varnothing$ and $x=w-\varepsilon u \in\left(E \boxminus \varepsilon B_{X}\right)+\varepsilon B_{X}=E_{\varepsilon}$. This proves that $E=E_{\varepsilon}^{\prime}$, i.e., $E$ is exactly $\varepsilon$-regular.

Conversely, let us assume $E$ is exactly $\varepsilon$-regular. We first observe that $E$ is solid: given $x \in E$ we can find $w \in E \boxminus \varepsilon B_{X}$ and $b \in B_{X}$ such that $x=w+\varepsilon b$ and for any sequence $\left(\varepsilon_{n}\right)$ in $(0, \varepsilon)$ with limit $\varepsilon$ we have $x=\lim x_{n}$ with $x_{n}:=w+\varepsilon_{n} b \in \operatorname{int}(E)$. Then $\operatorname{bd}(F)=\operatorname{bd}(E)$ by [14, Lemma 4.2]. Thus $F$ is philonormal by Proposition 2.19. Now let us assume that the closed set $E$ is one-sided and $X$ is smooth and strictly convex. Let $x \in \operatorname{bd}(F)$ and $u \in N_{m}(F, x) \cap B_{X}$. Then $x-\varepsilon b \in E \boxminus \varepsilon B_{X}$ for some $b \in B_{X}$ and $x-\varepsilon b+$ $\varepsilon B_{X} \subset E$. Since $B_{X}$ is smooth, there exists an open half-space $H$ which is included in $I\left(x-\varepsilon b+\varepsilon B_{X}, x\right) \subset I(E, x)=X \backslash T(F, x)$. In fact, as easily seen, $H=\{w \in X:\langle J(b), w\rangle<0\}$, where $J$ is the duality mapping, i.e. the derivative of half the square of the norm. Since $E$ is one-sided, its tangent cone $T(E, x)$ at $x$ is contained in some closed half-space; thus this half-space contains $H$, hence coincides with $\mathrm{cl}(H)$ and $H=I(E, x)=I(\operatorname{int}(E), x)$. It follows that $T(F, x)=X \backslash I(\operatorname{int}(E), x)=X \backslash H=-\operatorname{cl}(H)$. Now, as $u \in$ $N_{m}(F, x)$, we have $J(u) \in N(F, x)$ i.e. $\langle J(u), w\rangle \geq 0$ for each $w \in T(F, x)$. Therefore, by the Farkas lemma, $J(u)=-\lambda J(b)$ for some $\lambda \geq 0$. Since $X$ is strictly convex, $J$ is injective and we have $u=-\lambda b$. Since $x \in \operatorname{bd}(E)$ and $x \in x-\varepsilon b+\varepsilon B_{X} \subset E$, we deduce that $\|b\|=1$, whence, as $u \in B_{X}, \lambda \leq 1$. So we conclude that

$$
\begin{aligned}
\operatorname{int}\left(x+\varepsilon u+\varepsilon\|u\| B_{X}\right)=\operatorname{int}(x-\varepsilon \lambda b & \left.+\varepsilon \lambda B_{X}\right) \\
& \subset \operatorname{int}\left(x-\varepsilon b+\varepsilon B_{X}\right) \subset \operatorname{int}(E),
\end{aligned}
$$

which shows that $\operatorname{int}\left(x+\varepsilon u+\varepsilon\|u\| B_{X}\right) \cap \operatorname{cl}(X \backslash E)=\emptyset$ or $u \in N_{\varepsilon}(F, x)$. Thus $F$ is $\varepsilon$-weakly convex. 
Corollary 2.27. A closed convex subset with nonempty interior of a finite dimensional Euclidean space $X$ is $\varepsilon$-regular if and only if the closure of its complement is $\varepsilon$-weakly convex.

Proof. Such a set $E$ is known to be solid and proximinal. Since $X$ is smooth and strictly convex and since any proximally smooth subset is philonormal, $E$ is (exactly) $\varepsilon$-regular if and only if $F:=\operatorname{cl}(X \backslash E)$ is $\varepsilon$-proximally smooth or, equivalently (as $X$ is finite dimensional), $\varepsilon$-weakly convex.

\section{Regularization of functions}

Let us first observe that when $E$ (resp. $B$ ) is the epigraph of a function $f$ (resp. $b$ ) with values in $\mathrm{R} \cup\{+\infty\}$, then $\operatorname{cl}(E+B)$ is the epigraph of the lower semicontinuous hull $f \bar{\square} b$ of the function $f \square b$ obtained by infimal convolution of $f$ and $b$ :

$$
(f \square b)(x):=\inf _{w \in X}(f(x-w)+b(w)),
$$

with the convention $(+\infty)+(-\infty)=+\infty$. The set $E \boxminus B$ is the epigraph of the function $f \boxminus b$ obtained by deconvolution of $f$ by $b$ (see [4], [22], [23], [32], [33]) in the following way:

$$
(f \boxminus b)(x):=\sup _{w \in X}(f(x+w)-b(w)),
$$

with $s-t:=-(t+(-s))$ for $s, t \in \overline{\mathrm{R}}:=\mathrm{R} \cup\{+\infty,-\infty\}$. Let us prove this latter fact (which is less classical than the first one) by means of the following equivalences:

$$
\begin{aligned}
(x, r) \in E \boxminus B & \Leftrightarrow(x, r)+B \subset E \\
& \Leftrightarrow f(x+w) \leq r+s \quad \forall(w, s) \in B \\
& \Leftrightarrow r \geq \sup _{w \in \operatorname{dom} b}(f(w+x)-b(w)),
\end{aligned}
$$

using the observation that for $r \in \mathrm{R}, s, t \in \mathrm{R} \cup\{+\infty\}$, the relation $r \geq s-t$ amounts to $t=+\infty$ or $t<+\infty$ and $r \geq s-t$. Let us observe that the same results hold when $B$ is the strict epigraph $\operatorname{epi}_{s}(f)$ of the function $b$.

Thus, given an extended real-valued function $b: X \rightarrow \overline{\mathrm{R}}, b \neq \equiv+\infty$, we define the $b$-regularized function associated with a lower semicontinuous function $f: X \rightarrow \overline{\mathrm{R}}$ as the function $f_{b}: X \rightarrow \overline{\mathrm{R}}$ whose epigraph is epi $\left(f_{b}\right)=$ $\operatorname{epi}(f)_{\mathrm{epi}(b)}$. Since

$$
\operatorname{epi}(f)_{\text {epi }(b)}=\operatorname{cl}((\operatorname{epi}(f) \boxminus \operatorname{epi}(b))+\operatorname{epi}(b))=\operatorname{epi}((f \boxminus b) \bar{\square} b),
$$


one has $f_{b}=(f \boxminus b) \bar{\square}$.

Let us point out two other links between regularization of sets and regularization of functions. First, we observe that when $f$ is the indicator function $\iota_{E}$ of some subset $E$ of $X$ given by $\iota_{E}(x):=0$ for $x \in E, \iota_{E}(x):=+\infty$ when $x \in X \backslash E$, and when $b=\iota_{B}$ for some subset $B$ of $X$, then $f_{b}=\iota_{E_{B}}$. Now, using Proposition 2.2 (c) with a subset $B$ of $X \times \mathbf{R}, C:=\{0\} \times \mathbf{R}_{+}$, $A=B+C=\operatorname{epi}(b), E:=\operatorname{epi}(f)$, we get epi $(f)_{\text {epi }(b)}=E_{B}$. We will use this latter fact in the special case $B$ is a ball in $X \times \mathrm{R}$.

In the first part of this section we shall state some results on regularization of functions that parallel those of Section 2 on regularization of sets. In particular, according to Proposition 2.2, the following properties hold; here the recession function $0^{+} f$ of a function $f$ is the function $f \boxminus f$, so that a function $g$ satisfies $g \geq 0^{+} f$ if and only if epi $f+$ epi $g \subset$ epi $f$.

PROPOSITION 3.1.

(a) If $f \leq g$, then one has $f_{b} \leq g_{b}$.

(b) If $\bar{b} \leq a \leq b$, where $\bar{b}$ is the lower semicontinuous hull of $b$, then $f_{a} \leq f_{b}$.

(c) If $b \bar{\square} c \leq a \leq b \square c$, then one has $f_{b} \leq f_{a}$.

(d) If $b \square c \leq a \leq b \square c$, with $a \leq b$, $c \geq 0^{+} f$, then one has $f_{b}=f_{a}$.

(e) If $w \in X, r \in \mathbf{R}$, and $f^{w, r}: X \rightarrow \overline{\mathrm{R}}$ denotes the function defined by $f^{w, r}(x)=f(w+x)-r$, then one has $\left(f^{w, r}\right)_{b}=\left(f_{b}\right)^{w, r} ;$ if $c=b^{w, r}$, then $f_{c}=f_{b}$.

(f) If $\lambda>0$ and $f^{\lambda}: X \rightarrow \overline{\mathrm{R}}$ denotes the function defined by $f^{\lambda}(x)=$ $\lambda f\left(\lambda^{-1} x\right)$, then one has $\left(f^{\lambda}\right)_{b}=\left(f_{b^{\lambda-1}}\right)^{\lambda}$.

(g) For any functions $f$, $g$, one has $f_{b} \boxminus g_{b} \leq f \boxminus g$.

(h) If $f$ and $b$ are convex, then $f_{b}$ is convex.

In general, one has $f \leq f_{b}$, so that our regularized functions are upper approximations. Functions for which equality holds will be called $b$-regular:

Definition 3.2. A lower semicontinuous function $f: X \rightarrow \overline{\mathrm{R}}$ is said to be $b$-regular if $f_{b}=f$.

According to this definition, a function $f$ is $b$-regular if and only if epi $(f)$ is epi $(b)$-regular. From Proposition 2.5, the following characterization of $b$ regular functions follows:

Proposition 3.3. A lower semicontinuous function $f$ is $b$-regular if and only if there exists a function $g: X \rightarrow \overline{\mathrm{R}}$ such that $g \bar{\square} b=f$. 
Proof. If $f$ is $b$-regular then, for $g=f \boxminus b$, one has $g \bar{\square} b=(f \boxminus b) \bar{\square}$ $b=f_{b}=f$. Conversely, if there is a function $g$ satisfying $g \bar{\square} b=f$ then

$$
\operatorname{epi}(f)=\operatorname{epi}(g \bar{\square} b)=\operatorname{cl}(\operatorname{epi}(g)+\operatorname{epi}(b)),
$$

whence, by Proposition 2.5, epi $(f)$ is epi $(b)$-regular.

In view of the preceding proposition, the function $f_{b}$ is $b$-regular. As a consequence, one has

COROLlary 3.4. The b-regularization operator is idempotent, that is, $\left(f_{b}\right)_{b}=f_{b}$.

COROLlary 3.5. $f_{b}$ is the smallest $b$-regular majorant of $f$.

Using Proposition 3.1 (c), one easily gets the next consequence.

Corollary 3.6. If $b \bar{\square} c \leq a \leq b \square c$ and if $f$ is a-regular, then $f$ is $b$-regular, too.

In the same way as in the case of regularization of sets we mostly considered balls as the regularizing sets, in the case of functions we shall concentrate on the basic regularizing function $b: X \rightarrow \overline{\mathrm{R}}$ defined by

$$
b(x)=-\sqrt{1-\|x\|^{2}} \quad \text { if }\|x\| \leq 1,+\infty \text { otherwise. }
$$

We shall also consider the functions $b^{\varepsilon}: X \rightarrow \overline{\mathrm{R}}$ given by $b^{\varepsilon}(x)=\varepsilon b\left(\varepsilon^{-1} x\right)$. Using the preceding observations and noting that epi $(b)=B_{X \times \mathbf{R}}+\{0\} \times \mathbf{R}_{+}$, we see that, for any function $f: X \rightarrow \overline{\mathrm{R}}$, we have epi $\left(f_{b^{\varepsilon}}\right)=(\operatorname{epi}(f))_{\varepsilon B_{X \times \mathrm{R}}}$, $B_{X \times \mathrm{R}}$ denoting the unit ball of the space $X \times \mathrm{R}$ normed by $\|(x, r)\|_{X \times \mathrm{R}}=$ $\sqrt{\|x\|^{2}+r^{2}}$. We shall use the abbreviated notation $f_{\varepsilon}:=f_{b^{\varepsilon}}$ and the terms $\varepsilon$ regularized and $\varepsilon$-regular instead of $b^{\varepsilon}$-regularized and $b^{\varepsilon}$-regular, respectively. Thus, using also the abbreviated notation of Section 2, one can write epi $\left(f_{\varepsilon}\right)=$ $(\text { epi }(f))_{\varepsilon}$; the $\varepsilon$-regularization operation for functions is therefore equivalent to the $\varepsilon$-regularization of their epigraphs, and a function is $\varepsilon$-regular if and only if its epigraph is $\varepsilon$-regular. In particular, it follows from Corollary 2.13 that any concave lower semicontinuous function is $\varepsilon$-regular for any $\varepsilon>0$.

Propositions 2.10 and 3.1 yield the following basic properties of the $\varepsilon$ regularization operation:

Proposition 3.7.

(a) If $f \leq g$, then one has $f_{\varepsilon} \leq g_{\varepsilon}$.

(b) If $\varepsilon \geq \varepsilon^{\prime}>0$ then $f_{\varepsilon^{\prime}} \leq f_{\varepsilon}$.

(c) If $w \in X, r \in \mathrm{R}$, and $f^{w, r}:=f(w+\cdot)-r$, then one has $\left(f^{w, t}\right)_{\varepsilon}=$ $\left(f_{\varepsilon}\right)^{w, t}$. 
(d) If $\lambda>0$ then $\left(f^{\lambda}\right)_{\varepsilon}=\left(f_{\frac{\varepsilon}{\lambda}}\right)^{\lambda}$.

(e) $f_{\varepsilon} \boxminus g_{\varepsilon} \leq f \boxminus g$.

(f) $f$ is $\varepsilon$-regular if and only if there exists a function $g: X \rightarrow \overline{\mathrm{R}}$ such that $g \bar{\square} b^{\varepsilon}=f$.

(g) $\left(f_{\varepsilon}\right)_{\varepsilon}=f_{\varepsilon}$.

(h) $f_{\varepsilon}$ is the smallest $\varepsilon$-regular majorant of $f$.

(i) If $\varepsilon \geq \varepsilon^{\prime}>0$ and $f$ is $\varepsilon$-regular then it is $\varepsilon^{\prime}$-regular, too.

Similarly, Corollary 2.11 yields a property which has some similarity with the semi-group property of the Moreau regularization; it might be related to the so-called max-plus algebra.

Corollary 3.8. $\left(f_{\varepsilon}\right)_{\varepsilon^{\prime}}=f_{\varepsilon \vee \varepsilon^{\prime}}$.

The next result is a direct consequence of Corollary 2.12.

COROLlary 3.9. The lower semicontinuous hull of the pointwise infimum of a family of $\varepsilon$-regular functions is $\varepsilon$-regular.

We observe that this regularization process has some interest only for those functions whose epigraphs have a nonempty interior, i.e. those functions which are bounded above on some open subset; this is the case when the domains of the functions have the Baire property. In fact, by applying Corollary 2.16 to epigraphs, one obtains the following result:

Proposition 3.10. $f_{\varepsilon} \not \equiv+\infty$ for some $\varepsilon>0$ if, and only if, $f$ is bounded from above on some open set.

Since the $\varepsilon$-regularization of sets preserves convexity, the $\varepsilon$-regularization of functions preserves convexity, too.

PROPOSITION 3.11. If $f$ is convex then $f_{\varepsilon}$ is convex for every $\varepsilon$. Conversely, if all the functions $f_{\varepsilon}$ are convex and $f$ has a solid epigraph then $f$ is convex.

Proof. This is an immediate consequence of Proposition 2.17.

The following result characterizes $\varepsilon$-regularity of convex functions:

Proposition 3.12. A lower semicontinuous proper convex function $f$ is $\varepsilon$-regular if and only if $f^{*}-\varepsilon \sqrt{1+\|\cdot\|_{X^{*}}^{2}}$ is convex and $w^{*}$-lower semicontinuous.

Proof. If $f$ is $\varepsilon$-regular then $\left(f \boxminus b^{\varepsilon}\right) \bar{\square} b^{\varepsilon}=f$, whence $\left(f \boxminus b^{\varepsilon}\right)^{*}+$ $\left(b^{\varepsilon}\right)^{*}=f^{*}$, so that $f^{*}-\varepsilon \sqrt{1+\|\cdot\|_{X^{*}}^{2}}=f^{*}-\left(b^{\varepsilon}\right)^{*}$ is the convex function $(f \boxminus$ $\left.b^{\varepsilon}\right)^{*}$. Conversely, if $f^{*}-\varepsilon \sqrt{1+\|\cdot\|_{X^{*}}^{2}}$ is convex and $w^{*}$-lower semicontinuous, 
then, one has $f^{*}-\varepsilon \sqrt{1+\|\cdot\|_{X^{*}}^{2}}=g^{*}$ for some lower semicontinuous proper convex function $g$. It follows that $f^{*}=g^{*}+\varepsilon \sqrt{1+\|\cdot\|_{X^{*}}^{2}}=g^{*}+\left(b^{\varepsilon}\right)^{*}=$ $\left(g \bar{\square} b^{\varepsilon}\right)^{*}$, and hence $f=g \bar{\square} b^{\varepsilon}$. Therefore, by Proposition 3.7 (f), $f$ is $\varepsilon$-regular.

We say that a function $f$ is $\varepsilon$-regular at $x \in X$ if $f_{\varepsilon}(x)=f(x)$. We recall that the proximal subdifferential of $f$ at $x$ is the set of $x^{*} \in X^{*}$ such that $\left(x^{*},-1\right) \in J\left(N_{m}(E,(x, f(x)))\right.$, where $E$ is the epigraph of $f$ and $J$ is the duality mapping of $X \times \mathbf{R}$.

Proposition 3.13. Suppose $X$ is reflexive and $f$ is a weakly lower semicontinuous function. Then $f$ is $\varepsilon$-regular at $x \in \operatorname{dom} f$ for some $\varepsilon>0$ if and only if the metrically normal cone to the epigraph of the lower semicontinuous hull of $-f$ does not reduce to the origin. In particular, this occurs when $f$ is continuous and the proximal subdifferential at $x$ of $-f$ is nonempty.

Proof. Let $E$ be the epigraph of $f$. Clearly, $x_{f}:=(x, f(x))$ is a boundary point of $E$. Since $\operatorname{cl}((X \times \mathrm{R}) \backslash E)$ is the hypograph of the upper semicontinuous hull of $f$, its image by the symmetry $(x, r) \mapsto(x,-r)$ is the epigraph of the lower semicontinuous hull of $-f$. Our assumptions ensure that for any $\varepsilon>0$ the set $E_{\varepsilon}^{\prime}:=\left(E \boxminus \varepsilon B_{X \times \mathrm{R}}\right)+\varepsilon B_{X \times \mathrm{R}}$ is weakly closed, hence it is the epigraph of $f_{\varepsilon}$. It follows from Proposition 2.19 that $x_{f} \in E_{\varepsilon}^{\prime}$ if and only if $N_{\varepsilon}\left(\operatorname{cl}((X \times \mathrm{R}) \backslash E), x_{f}\right)$ does not reduce to the origin.

Let us recall [51] that an extended real-valued function $f$ on a finite dimensional Euclidean space is said to be $\rho$-convex, with $\rho \in \mathbf{R}$, if for every $x_{1,} x_{2} \in \operatorname{dom} f$ and $\lambda \in[0,1]$ one has

$$
f\left((1-\lambda) x_{1}+\lambda x_{2}\right) \leq(1-\lambda) f\left(x_{1}\right)+\lambda f\left(x_{2}\right)-\rho \lambda(1-\lambda)\left\|x_{1}-x_{2}\right\|^{2} .
$$

Proposition 3.14. Let $f$ be a lower semicontinuous convex function on a finite dimensional Euclidean space and assume that $\operatorname{dom} f$ has a nonempty interior. Then $f$ is $\varepsilon$-regular if and only if the hypograph of $f$, the smallest upper semicontinuous majorant of $f$, is $\varepsilon$-weakly convex. Hence, if $-\underline{f}$ is $-\frac{1}{2 \varepsilon}$-convex then $f$ is $\varepsilon$-regular.

Proof. The first part of the statement is an immediate consequence of Corollary 2.27. The second part follows from [51, Prop. 5.17].

In the remaining of this section, we consider smoothness questions. We first assume that $X$ is the Euclidean space $\mathrm{R}^{n}$, the basic regularizing function $b$ being the one defined by $b(x)=-\sqrt{1-\|x\|^{2}}$ if $\|x\| \leq 1,+\infty$ otherwise, $\|\cdot\|$ denoting the Euclidean norm. 
Proposition 3.15. If $f: \mathbf{R}^{n} \rightarrow \overline{\mathrm{R}}$ is convex then, for every $\varepsilon, f_{\varepsilon}$ is differentiable on the interior of its domain.

Proof. Since epi $(f)$ is convex and the unit ball $B_{\mathrm{R}^{n} \times \mathrm{R}}$ corresponding to the norm $\|\cdot\|_{\mathrm{R}^{n} \times \mathrm{R}}$ given by $\|(x, r)\|_{\mathrm{R}^{n} \times \mathrm{R}}=\sqrt{\|x\|^{2}+r^{2}}$ is smooth, by Proposition 2.22, for every $\varepsilon>0$ the set epi $\left(f_{\varepsilon}\right)=\operatorname{epi}(f)_{\varepsilon B_{\mathrm{R}^{n} \times \mathrm{R}}}$ is smooth, too, which implies that $f_{\varepsilon}$ is differentiable on the interior of its domain.

COROLlARY 3.16. Every E-regular convex function on $\mathrm{R}^{n}$ is differentiable on the interior of its domain.

Now let us consider the question of firm smoothness, i.e. of Fréchet differentiability. We need a piece of terminology. A modulus is a nondecreasing function $\alpha: \mathbf{R}_{+} \rightarrow \bar{R}_{+}$such that $\alpha(0)=0$ and is continuous at 0 . A gage is a nondecreasing function $\gamma: \mathbf{R}_{+} \rightarrow \bar{R}_{+}$which is positive on $\mathbf{P}:=(0,+\infty)$ and satisfies $\gamma(0)=0$. We say that a lower semicontinuous proper convex function $f: X \rightarrow \overline{\mathrm{R}}$ is essentially firmly smooth if it is Fréchet differentiable at each point of the domain of $\partial f$. This notion seems to be close to the concept of essential smoothness [5]. It implies that the domain of $\partial f$ coincides with the interior of the domain of $f$. A convex function $g: Y \rightarrow \overline{\mathrm{R}}$ is said to be essentially firmly convex if for any $y$ in the domain of $\partial g$ there exists a gage $\gamma$ and some $x \in \partial g(y)$ such that

$$
g(y+z) \geq g(y)+\langle x, z\rangle+\gamma(\|z\|) \quad \forall z \in Y .
$$

When this inequality holds, we say that $\gamma$ is is a gage of firm convexity of $g$ at $(y, x)$. A modulus $\alpha$ such that

$$
|f(x+w)-f(x)-\langle y, w\rangle| \leq \alpha(\|w\|)\|w\| \quad \forall w \in X
$$

will be called a modulus of (firm or Fréchet) differentiability of $f$ at $x$. We will make use of the following characterization which is similar to [53, Theorem 2.1], [3, Prop. 3.2], [54, Thm. 3.5.5].

LEMMA 3.17. A lower semicontinuous proper convex function $f$ on the Banach space $X$ is essentially firmly smooth if and only if its conjugate $g:=f^{*}$ on $Y:=X^{*}$ is essentially firmly convex. Moreover, $\gamma$ is a gage of firm convexity of $g$ at $(y, x)$ if and only if the function $\alpha: t \mapsto t^{-1} \gamma^{*}(t)$ is a modulus of differentiability of $f$ at $x$.

Theorem 3.18. Let $X$ be a reflexive Banach space and let $b: X \rightarrow$ $\mathrm{R} \cup\{+\infty\}$ be a lower semicontinuous, proper, coercive and essentially firmly smooth convex function. Then, for any lower semicontinuous proper convex function $f$ on $X$ the regularized function $f_{b}$ is essentially firmly smooth. In particular it is of class $\mathrm{C}^{1}$ on the interior of its domain. 
Proof. We first observe that since $b$ is coercive, $b$ and $f \boxminus b$ are convex, weakly semicontinuous and $X$ is reflexive, the regularization is exact: for any $x \in \operatorname{dom} f_{b}$ there exists $u, v \in X$ such that $u+v=x$ and $f_{b}(x)=$ $(f \boxminus b)(u)+b(v)$. Moreover, by a simple and well-known argument, for any $y \in \partial f_{b}(x)$ one has $y \in \partial(f \boxminus b)(u)$ and $y=b^{\prime}(v)$; moreover $x \in \partial f_{b}^{*}(y)$ and $x=u+v$ with $u \in \partial(f \boxminus b)^{*}(y)$ and $v \in \partial b^{*}(y)$. If $\gamma$ is a gage of firm convexity of $b^{*}$ at $(y, v)$, then $\gamma$ is a gage of firm convexity of $f_{b}^{*}$ at $(y, x)$. Therefore $f_{b}$ is firmly differentiable at $x$. The last assertion follows from the observations above and from a classical fact about continuity of the derivative of convex Fréchet differentiable functions $([9$, p. 86]).

\section{An algebraic point of view}

It is our purpose to establish a connexion between some notions of regularization and the concepts of closure and duality; we refer to [10], [27]-[31], [35], [39], [46] for generalities about dualities and polarities.

In the following statement, given two maps $D_{1}, D_{2}: G \rightarrow F$ with values in an ordered space $F$, we write $D_{1} \leq D_{2}$ if for any $g \in G$ we have $D_{1}(g) \leq$ $D_{2}(g)$. If $G$ is also an ordered space, we say that $D: F \rightarrow G$ is a homotone (resp. antitone) mapping if $D\left(f_{1}\right) \leq D\left(f_{2}\right)$ (resp. $D\left(f_{1}\right) \geq D\left(f_{2}\right)$ ) whenever $f_{1} \leq f_{2}$.

Lemma 4.1. Given two complete lattices $F, G$ and a homotone mapping $D: F \rightarrow G$, there exists a greatest homotone mapping $D^{\prime}: G \rightarrow F$ such that $D^{\prime}(D(f)) \leq f$ for any $f \in F$. It is given by

$$
D^{\prime}(g)=\inf \{h \in F: D(h) \geq g\} .
$$

Proof. The result is elementary. It also follows from [39] Lemma 3.2, providing $F$ with the reverse order.

The case when $D$ is a morphism of inf-lattices, i.e. $D$ satisfies the relation

$$
D\left(\inf _{i \in I} f_{i}\right)=\inf _{i \in I} D\left(f_{i}\right)
$$

for any family $\left(f_{i}\right)_{i \in I}$ of $F$ is of special interest. In such a case, $D$ can be considered as a duality in the sense of [31] when $G$ is endowed with the reverse order, so that $D^{\prime}$ is the reverse duality and the roles of $D$ and $D^{\prime}$ are symmetric (when viewed as dualities). More precisely, $D$ is obtained from $D^{\prime}$ by setting

$$
D(f)=\sup \left\{h \in G: D^{\prime}(h) \leq f\right\} .
$$


LeMmA 4.2. Let $X$ be a vector space and let $b \in F:=\overline{\mathrm{R}}^{X}$ be a function with nonempty domain. Then, the reverse mapping $D^{\prime}$ associated with the mapping $D: f \mapsto f \square b$ is the mapping $D^{\prime}: F \rightarrow F$ given by $D^{\prime}(g)=g \boxminus b$ with

$$
(g \boxminus b)(x):=\sup _{w \in \operatorname{dom} b}(g(w+x)-b(w)) .
$$

If $X$ is a normed vector space, if $\bar{F}$ is the set of lower semicontinuous functions on $X$ and if $\bar{D}: \bar{F} \rightarrow \bar{F}$ is given by $\bar{D}(f):=f \bar{\square} b$, then the reverse mapping of $\bar{D}$ is also $D^{\prime}: g \mapsto g \boxminus b$.

Proof. Clearly, $D$ and $D^{\prime}$ are homotone, hence antitone when the order is reversed on one side. Moreover, $D: f \mapsto f \square b$ satisfies relation (3), so that it can be considered as a duality. Let us show that $D^{\prime}$ given by relation (2) satisfies $D^{\prime}(g)=g \boxminus b$ by noting the following equivalences:

$$
\begin{aligned}
h \square b \geq g & \Leftrightarrow \forall x \in X, \forall w \in \operatorname{dom} b, h(x)+b(w) \geq g(w+x) \\
& \Leftrightarrow \forall x \in X, h(x) \geq \sup _{w \in \operatorname{dom} b}(g(w+x)-b(w)) .
\end{aligned}
$$

When $g, h \in \bar{F}$, we observe that the relation $h \bar{\square} b \geq g$ is equivalent to the relation $h \square b \geq g$, hence to $h \geq g \boxminus b$ by what precedes.

Given a duality $D$ and its reverse duality $D^{\prime}$, one is led to introduce the classes

$$
\begin{aligned}
F_{D} & :=\left\{f \in F: D^{\prime}(D(f))=f\right\}=\left\{f \in F: D^{\prime}(D(f)) \geq f\right\}, \\
G_{D^{\prime}} & :=\left\{g \in G: D\left(D^{\prime}(g)\right)=g\right\}=\left\{g \in G: D\left(D^{\prime}(g)\right) \leq g\right\},
\end{aligned}
$$

in view of the fact that $D \circ D^{\prime}$ is a closure operator on $G$ and $D^{\prime} \circ D$ is a closure operator for the reverse order on $F$.

Taking for $F$ and $G$ the power set of a vector space $X$, what precedes can be transcribed for the operations $D: E \mapsto E+B$ and $D^{\prime}: E \mapsto E \boxminus B$ where the Minkowski difference $E \boxminus B$ is given by

$$
E \boxminus B:=\{x \in X: x+B \subset E\}
$$

These set theoretical operations correspond to the preceding ones through the lattice isomorphisms

$$
\begin{aligned}
E & \mapsto \iota_{E}, \\
f & \mapsto E_{f},
\end{aligned}
$$


where $E_{f}$ is the epigraph of $f$ and where $\iota_{E}$ is the indicator function of $E$ given by $\iota_{E}(x)=0$ if $x \in E, \iota_{E}(x)=+\infty$ if $x \in X \backslash E$. Since

$$
\begin{aligned}
& \left(\bigcup_{i \in I} E_{i}\right)+B=\bigcup_{i \in I}\left(E_{i}+B\right), \\
& \bigcap_{i \in I}\left(E_{i} \boxminus B\right)=\left(\bigcap_{i \in I} E_{i}\right) \boxminus B,
\end{aligned}
$$

reversing the order in the left hand sides of these relations we get polarities in the sense of Birkhoff [10] and others.

Convergence results

Let us first deal with the question of convergence as $t \rightarrow 0_{+}$of the family $\left(E_{t}\right)_{t>0}$ of sets associated with some closed subset $E$ of $X$. Here $B$ is the closed unit ball of $X$ and for a subset $E$ of $X$ we set

$$
E_{t}:=\operatorname{cl}((E \boxminus t B)+t B) \quad t>0 .
$$

We first observe that the containement $E_{t} \subset E$ implies that

$$
\limsup _{t \rightarrow 0_{+}} E_{t} \subset E,
$$

and we state a simple result:

Proposition 4.3. Suppose $E$ is solid. Then $\left(E_{t}\right)$ converges to $E$ for the Wijsman topology and the Painlevé-Kuratowski convergence.

Proof. By Corollary 2.15, $E=\operatorname{cl}\left(\bigcup_{t>0} E_{t}\right)$. According to Proposition 2.10 (b), the family $\left\{E_{t}\right\}_{t>0}$ is decreasing in $t$ with respect to inclusion; therefore, for every $x \in X$ one has $\lim _{t \rightarrow 0^{+}} d\left(x, E_{t}\right)=\inf _{t>0} d\left(x, E_{t}\right)=$ $d\left(x, \bigcup_{t>0} E_{t}\right)=d\left(x, \operatorname{cl}\left(\bigcup_{t>0} E_{t}\right)\right)=d(x, E)$.

$$
\begin{aligned}
\lim _{t \rightarrow 0^{+}} d\left(x, E_{t}\right)=\inf _{t>0} d\left(x, E_{t}\right)=d\left(x, \bigcup_{t>0} E_{t}\right) & =d\left(x, \operatorname{cl}\left(\bigcup_{t>0} E_{t}\right)\right) \\
& =d(x, E) .
\end{aligned}
$$

Thus $\left(E_{t}\right)$ converges to $E$ for the Wijsman topology. In particular, we have $E \subset \liminf \operatorname{in}_{t \rightarrow 0_{+}} E_{t}$ since for $x \in E$ we have $d\left(x, E_{t}\right) \rightarrow 0$ as $t \rightarrow 0_{+}$. Thus, by (5), we have Painlevé-Kuratowski convergence.

The study that follows has been inspired by [34, Prop. 1-5-2] for the case $X$ is locally compact and the space of subsets of $X$ is endowed with the "myope topology" (Fell topology) and [44, Thm. 3.2.1] for the case of convex subsets 
with nonempty interiors. Here we give a slight extension to a nonconvex case and we limit our study to our aim which is the convergence as $t \rightarrow 0_{+}$.

Proposition 4.4. Suppose $E$ is solid and weakly closed in $X$. Then $\left(E_{t}\right)$ converges to $E$ for the Mosco convergence.

Proof. We already know that $E \subset \liminf _{t \rightarrow 0_{+}} E_{t}$. Now, since $E_{t} \subset E$ for each $t>0$ and $E$ is weakly closed, we have w-lim $\sup _{t \backslash 0} E_{t} \subset E$. Thus $\left(E_{t}\right)$ converges to $E$ as $t \searrow 0$ for the Mosco convergence.

Now let us turn to the Pompeiu-Hausdorff convergence. Recall that the star (or stage) of a subset $E$ of $X$ is the set st $(E)$ of points of $E$ at which the set $E$ is starshaped:

$$
\operatorname{st}(E):=\{x \in X: \forall t \in[0,1], \forall e \in E, x+t(e-x) \in E\} .
$$

Proposition 4.5. Suppose $E$ is bounded and the interior of $\operatorname{st}(E)$ is nonempty. Then $\left(E_{t}\right) \rightarrow E$ for the Pompeiu-Hausdorff distance, with a linear speed of convergence: $\lim \sup _{t \searrow 0} t^{-1} d\left(E, E_{t}\right)<+\infty$.

More precisely, let $c$ be the infimum of the ratio $s / r$ where $(r, s)$ is a pair of positive numbers for which there exists some $a \in E$ such that $B(a, r) \subset \operatorname{st}(E)$ and $E \subset B(a, s)$. Then one has

$$
\limsup _{t \searrow 0} t^{-1} d\left(E, E_{t}\right) \leq c-1 .
$$

Note that when $E$ is convex $c$ is a measure of the nonsphericity of the set $E$.

Proof. Given $q>c$, we can find $a \in \operatorname{st}(E)$ and $r>0$ such that $B(a, r) \subset$ st $(E)$ and $E \subset B(a, q r)$. Let $t \in[0, r]$. For each $x \in E$ let us check that $y:=x+t r^{-1}(a-x)$ belongs to $E \boxminus t B$. In fact, for each $u \in B$ we have $y+t u=x+t r^{-1}(a+r u-x) \in E$ since $t r^{-1} \in[0,1]$ and $a+r u \in \operatorname{st}(E)$. Thus $y \in E \boxminus t B$. Now $\|y-x\| \leq t q$ and since $z:=y+q^{-1}(x-y) \in(E \boxminus t B)+t B$ we have $d\left(x, E_{t}\right) \leq\|x-z\| \leq t(q-1)$. Since this estimate is valid for any $x \in E$ and since $E_{t}$ is included in $E$, we get the result.

An extension to the case of unbounded subsets along the lines of [44] can be given under the assumption that the recession cone $E \boxminus E$ of $E$ has a nonempty interior. Another extension is as follows.

Proposition 4.6. Let $E$ be a closed subset of $X$ such that for each $p>0$ large enough the interior of $\operatorname{st}(E \cap p B)$ is nonempty. Then $\left(E_{t}\right) \stackrel{b}{\rightarrow} E$, i.e. $\left(E_{t}\right)$ converges to $E$ for the bounded-Hausdorff distance.

Proof. Given $p>0$, replacing $E$ by $E \cap p B$ in the preceding proof, we see that $d\left(E \cap p B,(E \cap p B)_{t}\right) \rightarrow 0$ as $t \searrow 0$. Since $(E \cap p B)_{t} \subset E_{t}$, the result follows. 
Now let us deal with convergence questions for functions. Since variational convergences are introduced by the means of various convergences for the epigraphs of the functions (see [6], [42], [45], [49] for instance), the preceding results readily entail convergence properties for these variational convergences. In particular, we have the following convergence results. Here we say that a function $f$ is solid if its epigraph is solid. Since the interior of the epigraph of a function is the strict epigraph of its upper semicontinuous hull, and since the epigraph of its lower semicontinuous hull is the closure of its epigraph, we get that $f$ is solid if and only if it coincides with the lower semicontinuous hull of its upper semicontinuous hull. This property occurs when $f$ is continuous on its domain and has a solid domain.

Proposition 4.7.

(a) Let $f$ be solid. Then $\left(f_{t}\right)$ epi-converges to $f$.

(b) Suppose moreover that $f$ is weakly lower semicontinuous. Then $\left(f_{t}\right)$ converges to $f$ for the Mosco convergence.

(c) If $f$ is a lower semicontinuous convex function which is continuous on the interior of its domain, then $\left(f_{t}\right) \stackrel{b}{\rightarrow} f$ i.e. $\left(f_{t}\right)$ converges to $f$ for the bounded-Hausdorff distance.

More specific results can also be given. Since the $\varepsilon$-regularizations of $f$ are majorants of $f$ and, according to Proposition 3.7 (b), they depend on $\varepsilon$ in a nondecreasing way, for every $x \in X$ one has

$$
\lim _{\varepsilon \rightarrow 0^{+}} f_{\varepsilon}(x)=\inf _{\varepsilon>0} f_{\varepsilon}(x) \geq f(x) .
$$

The next proposition shows that equality holds if $x$ is a continuity point of $f$.

PROPOSITION 4.8.

(a) If the epigraph of $f$ is solid, then $f$ is the lower semicontinuous hull of the function $x \mapsto \lim _{\varepsilon \rightarrow 0^{+}} f_{\varepsilon}(x)$.

(b) If $f$ is upper semicontinuous at $x \in \operatorname{int}(\operatorname{dom}(f))$ then

$$
\lim _{\varepsilon \rightarrow 0^{+}} f_{\varepsilon}(x)=\inf _{\varepsilon>0} f_{\varepsilon}(x)=f(x) .
$$

Proof. (a) If epi $(f)$ is solid then, by Corollary 2.15,

$$
\begin{aligned}
\operatorname{epi}(f) & =\operatorname{cl}(\operatorname{int}(\operatorname{epi}(f)))=\operatorname{cl}\left(\bigcup_{\varepsilon>0} \operatorname{epi}(f)_{\varepsilon}\right)=\operatorname{cl}\left(\bigcup_{\varepsilon>0} \operatorname{epi}\left(f_{\varepsilon}\right)\right) \\
& =\operatorname{cl}\left(\operatorname{epi}_{\varepsilon>0}\left(\inf _{\varepsilon} f_{\varepsilon}\right)\right) ;
\end{aligned}
$$

since $\operatorname{cl}\left(\operatorname{epi}\left(\inf _{\varepsilon>0} f_{\varepsilon}\right)\right)$ is the epigraph of the lower semicontinuous hull of $\inf _{\varepsilon>0} f_{\varepsilon}=\lim _{\varepsilon \rightarrow 0^{+}} f_{\varepsilon}$, the statement follows. 
(b) Let $r>f(x)$. Then, by upper semicontinuity, $(x, r) \in \operatorname{int}(\operatorname{epi}(f))$, whence, in view of Proposition 2.14, $(x, r) \in \operatorname{epi}(f)_{\varepsilon_{0}}=\operatorname{epi}\left(f_{\varepsilon_{0}}\right)$ for some $\varepsilon_{0}>0$. Therefore inf $\varepsilon_{\varepsilon>0} f_{\varepsilon}(x) \leq f_{\varepsilon_{0}}(x) \leq r$. Since $r>f(x)$ is arbitrary, this shows that $\inf _{\varepsilon>0} f_{\varepsilon}(x) \leq f(x)$, which, in view of (6), proves (7).

When $f$ is uniformly continuous, the convergence of $f_{\varepsilon}$ towards $f$ is uniform:

Proposition 4.9. Suppose $f$ is uniformly continuous. Then $f_{\varepsilon}$ converges uniformly to $f$ as $\varepsilon \rightarrow 0^{+}$.

Proof. Let $\mu$ be a modulus of uniform continuity of $f: \mu$ is a modulus (i.e. $\mu: \mathbf{R}_{+} \rightarrow \mathbf{R}_{+} \cup\{+\infty\}$ is nondecreasing, continuous at 0 , with $\mu(0)=0$ ) and satisfies $f(x)-f(y) \leq \mu(\|x-y\|)$ for any $x, y \in X$. Then, for any $x \in X$ one has

$$
\begin{aligned}
\left(f \boxminus b^{\varepsilon}\right)(x)=\sup _{w \in \varepsilon B_{X}}\left(f(w+x)-b^{\varepsilon}(w)\right) & \leq \sup _{w \in \varepsilon B_{X}}(f(x)+\mu(\|w\|)+\varepsilon) \\
& \leq f(x)+\mu(\varepsilon)+\varepsilon .
\end{aligned}
$$

Therefore, since $b^{\varepsilon}(0)=-\varepsilon$,

$$
\left(\left(f \boxminus b^{\varepsilon}\right) \square b^{\varepsilon}\right)(x)-f(x) \leq\left(f \boxminus b^{\varepsilon}\right)(x)+b^{\varepsilon}(0)-f(x) \leq \mu(\varepsilon) .
$$

Since $f_{\varepsilon} \geq f$, we get that $\sup _{x \in X}\left|f_{\varepsilon}(x)-f(x)\right| \leq \mu(\varepsilon)$.

An easy modification of the preceding proof shows that if $f$ is uniformly continuous on some subset $D$ of its domain then, for any $\alpha>0, f_{\varepsilon}$ converges uniformly to $f$ on $D \boxminus \alpha B_{X}$. In particular, if $f$ is uniformly continuous on $\operatorname{int}(\operatorname{dom}(f))$ then $f_{\varepsilon}$ converges uniformly to $f$ on compact subsets of $\operatorname{int}(\operatorname{dom}(f))$.

\section{Generalizations and comparisons with other regularization processes}

We observe that for any subsets $A, E$ of $X$ we have

$$
E \boxminus A=\left(E^{c}+(-A)\right)^{c},
$$

where $E^{c}:=X \backslash E$; in fact, $x \in(E \boxminus A)^{c}$ if and only if there exists some $a \in A$ such that $x+a \in E^{c}$. It follows from this observation that the following variant of our regularization

$$
E_{\lambda A, \mu B}:=(E \boxminus \lambda A)+\mu B \quad \lambda, \mu>0
$$


is related to the Benoist regularization of $E$ [8], [7]; this regularization is given by

$$
H \mapsto R_{\lambda C, \mu B}(H):=\left((H+\lambda C)^{c}+\mu B\right)^{c} .
$$

Thus,

$$
\begin{aligned}
E_{\lambda A, \mu B} & =\left(R_{\lambda C, \mu B}(H)\right)^{c}, \\
R_{\lambda C, \mu B}(H) & =\left(E_{\lambda A, \mu B}\right)^{c}
\end{aligned}
$$

with $H=E^{c}, C=-A: R_{\lambda C, \mu B}(H)$ is obtained by a passage to the complement before and after using the regularization (8) with $A:=-C$. In the case $B$ is symmetric, this amounts to taking the shrinking and the enlargement in a reverse order:

$$
R_{\lambda A, \mu B}(H)=(H+\lambda A) \boxminus \mu B .
$$

The effect is quite different. Moreover, when one takes for $E$ the epigraph of a function $f$, the complement $E^{c}$ is not the hypograph of $f$ but its strict hypograph. For attainment questions, this may introduce slight discrepancies, even if the result is not essentially different.

Moreover, in the case $A=B, \lambda=\mu$, the Benoist regularization of $E$ is larger than $E$ whereas our regularized set is smaller than $E$. The same observation is valid for the $\varepsilon$-convex hull $C_{\varepsilon}(E)$ of $E$ defined in [43] which is the special case of the Benoist regularization given by $C_{\varepsilon}(E)=R_{-U, U}(E)$ with $U=\frac{\varepsilon}{2} \operatorname{int}\left(B_{X}\right)$; in fact in [43] this process is defined in a general metric space. Let us note the following consequence about the families of regular sets; here we return to our regularization rather than to its variant (8) which avoids closure.

Proposition 5.1. When $A=B=C$ is symmetric and weakly compact, $E$ is weakly closed, the set $H:=E^{c}$ is Benoist regular in the sense that it coincides with its Benoist regularization if and only if $E$ is regular in our sense.

Proof. The set $H$ is Benoist regular if and only if

$$
H=\left((H+C)^{c}+B\right)^{c}=((E \boxminus A)+B)^{c}=\left(E_{B}\right)^{c},
$$

which is equivalent to $E:=H^{c}=E_{B}$.

Now let us compare our regularization with the one studied in [14]. There, for a subset $E$ of $X$ and $r>0$, the authors study the outer and the inner approximations given respectively (with a slight change of notation in order to avoid confusions) by

$$
\begin{aligned}
& E^{(r)}:=\{x \in X: d(x, E) \leq r\} \\
& E_{(r)}:=\left\{x \in X: d\left(x, \operatorname{cl}\left(E^{c}\right)\right) \geq r\right\} .
\end{aligned}
$$


Proposition 5.2. For any closed subset $E$ of $X$ and any $r>0$ one has $E_{r}=\left(E_{(r)}\right)^{(r)}$.

Proof. We first observe that $E_{(r)}=E \boxminus r B_{X}$. In fact, as already observed, $E \boxminus r B_{X}=E \boxminus r \operatorname{int}\left(B_{X}\right)$ the ball $B_{X}$ being solid; moreover one has $x+$ $r \operatorname{int}\left(B_{X}\right) \subset E$ if and only if $d\left(x, E^{c}\right) \geq r$.

Now let us prove that for any subset $F$ of $X$ one has $F^{(r)}=\operatorname{cl}\left(F+r B_{X}\right)$. The inclusion $\operatorname{cl}\left(F+r B_{X}\right) \subset F^{(r)}$ is obvious. Obviously $F_{r}^{\prime}:=F+r B_{X} \subset F^{(r)}$, so that $\operatorname{cl}\left(F_{r}^{\prime}\right) \subset F^{(r)}$. Conversely, let $w \in F^{(r)}$. For each $\varepsilon>0$, there exists $x_{\varepsilon} \in F$ such that $\left\|x_{\varepsilon}-w\right\| \leq d(w, F)+\varepsilon \leq r+\varepsilon$. Let us set $y_{\varepsilon}:=x_{\varepsilon}+\frac{r}{r+\varepsilon}\left(w-x_{\varepsilon}\right)$. Then $y_{\varepsilon} \in F+r B_{X}=F_{r}^{\prime}$ and

$$
\left\|y_{\varepsilon}-w\right\|=\left|\frac{r}{r+\varepsilon}-1\right|\left\|x_{\varepsilon}-w\right\| \leq \frac{\varepsilon}{r+\varepsilon}(r+\varepsilon)=\varepsilon .
$$

Thus $w \in \operatorname{cl}\left(F_{r}^{\prime}\right)$. The result is a consequence of the equality $F^{(r)}=\operatorname{cl}(F+$ $\left.r B_{X}\right)$ when one takes $F:=E_{(r)}=E \boxminus r B_{X}$.

In [14] the regularizing effect of the operations $E \mapsto E^{(r)}$ and $E \mapsto E_{(r)}$ is disclosed in terms of the aperture (or maximal angle) of the Clarke normal cone. The analytic version of the regularization $E \mapsto E^{(r)}$ is studied in [24] and [47] for the case of convex functions and in [14] essentially in the case of Lipschitz functions. A double process similar to the one we used (but with two different parameters, as in the Lasry-Lions [25] process) is also introduced in [14] and used in connection with Hamilton-Jacobi equations and invariance issues. In view of the behavior of our regularization process in the convex and in the concave cases, a double approximation of the function (resp. set) and of its opposite (resp. complement) would be of interest.

\section{REFERENCES}

1. Attouch, H., and Azé, D., Approximation and regularization of arbitrary functions in Hilbert spaces by the Lasry-Lions method, Ann. Inst. H. Poincaré 10 (1993), 289-312.

2. Aubin, J.-P., Mutational and Morphological analysis. Tools for shape evolution and morphogenesis, Birkhäuser, Boston (1999).

3. Azé, D., and Penot, J.-P., Uniformly convex and uniformly smooth convex functions, Ann. Fac. Sci. Toulouse Math. (6) 4 (1995), 705-730.

4. Azé, D., and Volle, M., Various continuity properties of the deconvolution (or epigraphical difference), Advances in Optimization, Proceedings, Lambrecht, 1991, Lecture Notes in Econom. and Math. Systems 382 (1992), 16-30.

5. Bauschke, H. H., Borwein, J. M., and Combettes, P.-L., Essential smoothness, essential strict convexity and Legendre functions in Banach spaces, Communications in Contempory Maths. 3 (2001), 615-647.

6. Beer, G., Topologies on Closed and Convex Sets, Kluwer, Dordrecht (1993). 
7. Benoist, J., Approximation and regularization of arbitrary sets; the finite dimensional case, Publications Univ. Limoges (1993).

8. Benoist, J., Approximation and regularization of arbitrary sets in finite dimension, Set-Valued Anal. 2 (1994), 95-115.

9. Benyamini, Y., and Lindenstrauss, J., Geometric Nonlinear Functional Analysis, Vol. 1, Colloquium Publications 48, Amer. Math. Soc. (2000).

10. Birkhoff, G., Lattice Theory, Amer. Math. Soc., Providence (1966).

11. Bony, J.-M., Principe du maximum, inégalité de Harnack et unicité du problème de Cauchy pour les opérateurs elliptiques dégénérées, Ann. Inst. Fourier 19 (1969), 277-304.

12. Borwein, J. M., and Tingley, D. W., On supportless convex sets, Proc. Amer. Math. Soc. 94 (1985), 471-476.

13. Bougeard, M., Penot, J.-P., and Pommellet, A., Towards minimal assumptions for the infimal convolution regularization, J. Approx. Theory 64 (1991), 245-270.

14. Clarke, F. H., Ledyaev, Yu. S., and Stern, R. J., Complements, approximations, smoothings and invariance properties, J. Convex Anal. 4 (1997), 189-219.

15. Clarke, F. H., Ledyaev, Yu. S., and Stern, R. J., Invariance, monotonicity and applications, Nonlinear Analysis, Differential Equations and Control, F. H. Clarke and R. J. Stern, (eds.), Kluwer, Dordrecht (1999), 207-305.

16. Clarke, F. H., Stern, R. J., and Wolenski, P. R., Proximal smoothness and the lower-C $\mathrm{C}^{2}$ property, J. Convex Anal. 2 (1995), 117-144.

17. Cornet, B., and Czarnecki, M.-O., Smooth representations of epi-Lipschitzian subsets of $\mathbf{R}^{n}$, Nonlinear Anal., Theory Methods Appl. 37A (1999), 139-160.

18. Cornet, B., and Czarnecki, M.-O., Smooth normal approximations of epi-Lipschitzian subsets of $\mathrm{R}^{n}$, SIAM J. Control Optim. 37 (1999), 710-730.

19. Cornet, B., and Czarnecki, M.-O., Approximation methods in equilibrium and fixed point theory. Proximally nondegenerate sets, Nguyen, Van Hien (ed.) et al., Optimization. Proceedings of the 9th Belgian-French-German conference, Namur, Belgium, September 7-11, 1998, Lect. Notes in Econom. and Math. Systems 481 (2000), 91-110.

20. Duboviskij, A. J., and Miljutin, A. A., Extremum problems in the presence of restrictions, U.S.S.R. Comput. Math. Phys. 5 (1965), 1-80; transl from Zh. Vychisl Mat. Fiz 5 (1965), 395-453.

21. Federer, H., Curvature measures, Trans. Amer. Math. Soc. 93 (1959), 418-491.

22. Hiriart-Urruty, J.-B., The deconvolution operation in convex analysis: an introduction, Cybernet. Systems Anal. 30 (1994), 555-560; translation from Kibernet. Sist. Anal. 4 (1994), 97-104.

23. Hiriart-Urruty, J.-B., and Mazure, M.-L., Formulations variationnelles de l'addition parallèle et de la soustraction parallèle d'opérateurs semi-définis positifs, C. R. Acad. Sci. Paris Sér. I Math. 302 (1986), 527-530.

24. Ioffe, A. D., Euler-Lagrange and Hamiltonian formalisms in dynamic optimization, Trans. Amer. Math. Soc. 349 (1997), 2871-2900.

25. Lasry, J.-M., and Lions, P.-L., A remark on regularization in Hilbert spaces, Israel J. Math. 55 (1986), 257-266.

26. Lemaréchal C., and Sagastizábal, C., Practical aspects of the Moreau-Yosida regularization: Theoretical preliminaries, SIAM J. Optim. 7 (1997), 367-385.

27. Martínez-Legaz, J.-E., and Singer, I., Dualities between complete lattices, Optimization 21 (1990), 481-508.

28. Martínez-Legaz, J.-E., and Singer, I., $\vee$-dualities and $\perp$-dualities, Optimization 22 (1991), 483-511.

29. Martínez-Legaz, J.-E., and Singer, I., *-dualities, Optimization 30 (1994), 295-315.

30. Martínez-Legaz, J.-E., and Singer, I., Dualities associated to binary operations on $\overline{\mathrm{R}}$, J. Convex Anal. 2 (1995), 185-209. 
31. Martínez-Legaz, J.-E., and Singer, I., Subdifferentials with respect to dualities, ZOR-Math. Methods Oper. Res. 42 (1995), 109-125.

32. Mazure, M.-L., Equations de convolution et formes quadratiques, Ann. Mat. Pura Appl. IV. Ser. 158 (1991), 75-97.

33. Mazure, M.-L., and Volle, M., Équations inf-convolutives et conjugaison de Moreau-Fenchel, Ann. Fac. Sci. Toulouse V. Sér., Math. 12 (1) (1991), 103-126.

34. Matheron, G., Random Sets and Integral Geometry, Wiley Series in Probability and Mathematical Statistics, New York (1975).

35. Pallaschke, D., and Rolewicz, S., Foundations of Mathematical Optimization. Convex Analysis without Linearity, Maths. and its Appl., vol. 388, Kluwer, Dordrecht (1998).

36. Penot, J.-P., A characterization of tangential regularity, Nonlinear Anal., Theory, Method \& Appl. 25 (1981), 625-643.

37. Penot, J.-P., On regularity conditions in mathematical programming, Math. Prog. Study 19 (1982), 167-199.

38. Penot, J.-P., Compact nets, filters and relations, J. Math. Anal. Appl. 93 (1983), 400-417.

39. Penot, J.-P., What is quasiconvex analysis?, Optimization 47 (2000), 35-110.

40. Penot, J.-P., On the minimization of difference functions, J. Global Optim. 12 (1998), 373-382.

41. Penot, J.-P., Proximal mappings, J. Approx. Theory 94 (1998), 203-221.

42. Penot, J.-P., and Zălinescu, C., Continuity of usual operations and variational convergences, Set-Valued Anal. 11 (2003), 225-256.

43. Perkal, J., Sur les ensembles $\varepsilon$-convexes, Colloq. Math. 4 (1956), 1-10.

44. Pichard, K., Equations différentielles dans les espaces métriques. Applications à l'évolution de domaines, $\mathrm{PhD}$ thesis, University of Pau, November 2001.

45. Rockafellar, R. T., and Wets, R. J.-B., Variational Analysis, Springer-Verlag, Berlin (1997).

46. Rubinov, A. M., Abstract Convexity and Global Optimization, Kluwer, Dordrecht (2000).

47. Seeger, A., Smoothing a nondifferentiable convex function: the technique of the rolling ball, Rev. Mat. Apl. 18 (1997), 45-60.

48. Singer, I., Abstract Convex Analysis, Wiley, New York (1997).

49. Sonntag, Y., and Zălinescu, C., Set convergences. An attempt of classification, Trans. Amer. Math. Soc. 340 (1993), 199-226.

50. Strömberg, T., On regularization in Banach spaces, Ark. Mat. 34 (1996), 383-406.

51. Vial, J.-Ph., Strong and weak convexity of sets and functions, Math. Oper. Res. 8 (1983), 231-259.

52. Volle, M., A formula on the subdifferential of the deconvolution of convex functions, Bull. Austral. Math. Soc. 47 (1993), 333-340.

53. Zălinescu, C., On uniformly convex functions, J. Math. Anal. Appl. 95 (1983), 344-374.

54. Zălinescu, C., Convex Analysis in General Vector Spaces, World Scientific, Singapore (2002).

DEPARTAMENT D'ECONOMIA I

D'HISTÒRIA ECONÒMICA

UNIVERSITAT AUTÒNOMA DE BARCELONA

08193 BELLATERRA

SPAIN

E-mail: JuanEnrique.Martinez@uab.es
LABORATOIRE DE MATHÉMATIQUES APPLIQUÉES

CNRS 2055

FACULTÉ DES SCIENCES

AV. DE L'UNIVERSITÉ

64000 PAU

FRANCE

E-mail: Jean-Paul.Penot@univ-pau.fr 